\title{
Esophageal Perforation after Anterior Cervical Spine Surgery
}

\author{
Sang-Bong Ko ${ }^{1}$, Jong-Beom Park ${ }^{2}$, Kyung-Jin Song ${ }^{3}$, Dong-Ho Lee ${ }^{4}$, \\ Seong-Wan Kim ${ }^{5}$, Young-Yul Kim², Taek-Soo Jeon ${ }^{6}$, Yoon Joo Cho ${ }^{2}$ \\ ${ }^{1}$ Department of Orthopaedic Surgery, Daegu Catholic University Medical Center, Daegu, Korea \\ ${ }^{2}$ Department of Orthopaedic Surgery, College of Medicine, The Catholic University of Korea, Seoul, Korea \\ ${ }^{3}$ Department of Orthopedic Surgery, Chonbuk National University Hospital, Jeonju, Korea \\ ${ }^{4}$ Department of Orthopaedic Surgery, Asan Medical Center, Seoul, Korea \\ ${ }^{5}$ Department of Orthopaedic Surgery, National Police Hospital, Seoul, Korea \\ ${ }^{6}$ Department of Orthopaedic Surgery, Barun Mind Hospital, Daejeon, Korea
}

Study Design: Retrospective case analyses.

Purpose: To investigate the causes, diagnosis, and management of esophageal perforation, depending on the time of diagnosis. Overview of Literature: To date, few studies have addressed these issues.

Methods: A total of seven patients were included in this study. The patients were classified into three groups based on esophageal perforation diagnosis time: intraoperative (diagnosed during surgery), perioperative (diagnosed within 30 days postoperatively), and delayed (diagnosed $>30$ days postoperatively) groups.

Results: In the intraoperative group ( $\mathrm{N}=2$ ), infectious spondylitis was the main cause of esophageal perforation. Anterior plate and screw removal, followed by posterior instrumentation, was performed. The injured esophagus was managed by omentum flap repair in one patient and primary repair in one patient. In the perioperative group ( $\mathrm{N}=2)$, revision surgery for infection and metal failure were the main causes of esophageal perforation. In both cases, food residue was drained on the third postoperative day. The injured esophagus was managed conservatively. In the delayed group $(\mathrm{N}=3)$, chronic irritation caused by metal failure was the main cause of esophageal perforation. In all patients, there was no associated infection. The anterior instrumentation was removed, and the two patients were treated by primary repair, and one patient was treated using sternocleidomastoid muscle flap. One patient in intraoperative group died of sepsis.

Conclusions: The main cause of intraoperative esophageal perforation was esophageal adhesions because of infectious spondylitis. However, perioperative and delayed esophageal perforations were caused by chronic irritation because of metal failure. Anterior plate and screw removal was necessary, and posterior instrumentation and fusion may be considered, depending on the fusion status.

Keywords: Esophageal perforation; Anterior surgery; Cervical spine

\section{Introduction}

Dysphagia, hematoma, vocal cord paralysis, dural injury,
Horner syndrome, and esophageal perforation are some of the postoperative complications of anterior cervical spine surgery. Of these, esophageal perforation is a rare

Received Dec 25, 2018; Revised Jan 12, 2019; Accepted Jan 21, 2019

Corresponding author: Jong-Beom Park

Department of Orthopaedic Surgery, Uijeongbu St. Mary’s Hospital, College of Medicine, The Catholic University of Korea, 271 Cheonbo-ro, Uijeongbu 11765, Korea

Tel: +82-31-820-3578, Fax: +82-31-847-3671, E-mail: spinepjb@catholic.ac.kr 
but fatal complication, with mortality rates between $6 \%$ and $34 \%$. Because of its rarity, with an incidence ranging from $0.2 \%$ to $3.4 \%[1,2]$, most spine surgeons have limited experience in the treatment of esophageal perforation. To date, only a few case studies have been reported in the literature. Herein, we report the causes, diagnosis, and management of esophageal perforation, depending on the time of diagnosis.

\section{Materials and Methods}

This retrospective study was approved by the Institutional Review Board of the Uijeongbu St. Mary's Hospital (eIRB UC18RESI0153) and informed consent was waived. In total, seven patients treated by seven different spine surgeons were analyzed. The demographic characteristics of the patients are detailed in Table 1, and all patient data were reviewed retrospectively. In all patients, primary cervical spine lesion, primary cervical surgery type, risk factors, predicted causes of esophageal injury, duration from first symptom to surgery, period from surgery to diagnosis, reason for diagnostic delay, diagnostic method, coinfection, identification of organism, treatment method, and treatment results were examined. Based on the time of esophageal perforation, the patients were categorized into the following groups: the intraoperative group (diagnosed intraoperatively), perioperative group (diagnosed within 30 days postoperatively), and delayed group (diagnosed $>30$ days postoperatively).

\section{Results}

\section{Intraoperative group (case 1)}

A 60-year-old male patient with no other risk factors was referred to the clinic with infectious spondylitis (C5-C6) without the identification of bacterial source (Fig. 1A-C). The initial surgery was performed with anterior cervical corpectomy and fusion with strut autograft and plate fixation (C4-C7) (Fig. 1D) and esophageal perforation was suspected because of severe adhesion between the esophagus and vertebral body during the primary surgery. Esophageal perforation was diagnosed by esophagography (Fig. 1E). Gastrostomy feeding and additional posterior fixation were performed (Fig. 1F). One month after the initial surgery, primary repair was performed for esophageal perforation, and the anterior implant was removed
(Fig. 1G). Postoperative esophagography showed no visible leakage (Fig. 1H). Esophagography at 1-year followup showed kyphosis correction and segmental fusion, without sequelae (Fig. 1I).

\section{Perioperative group (case 2)}

An otherwise healthy 53-year-old male patient was treated with anterior cervical discectomy and fusion (ACDF, C5C6-C7) (Fig. 2A) for cervical herniated disc. At 3 months, the patient developed a high-grade fever with complaints of dysphagia. Simple radiography revealed migrated anterior plate and screw (Fig. 2B). A diagnosis of postoperative infection was made, followed by implant removal and debridement (Fig. 2C). Food residues were drained at 3 days after the removal surgery (Fig. 2G). Esophagography (Fig. 2E, F) and secondary exploration (Fig. 2D) were performed to diagnose esophageal perforation. Esophageal perforation was healed post conservative treatment (Fig. 2H, I).

\section{Delayed group (case 3 )}

An otherwise healthy 60 -year-old male patient was treated for unilateral facet fracture and traumatic disc rupture at the $\mathrm{C} 6-\mathrm{C} 7$ level using $\mathrm{ACDF}$ with plate and cage (Fig. 3A). He responded well to the treatment and was asymptomatic for 32 months. At that time, a foreign material was extruded from his mouth during coughing. He brought the cage to the clinic (Fig. 3B). The plate was found to be loosened in the imaging study (Fig. 3C, D), and esophagography (Fig. 3E) and endoscopy (Fig. $3 \mathrm{~F})$ revealed that the implant had migrated to the inner esophagus. A diagnosis of esophageal perforation was made. Anterior floating plate removal was performed (Fig. $3 \mathrm{G}$ ), and esophageal perforation was reconstructed with a sternocleidomastoid flap. Cervical fusion (Fig. 3H) and a healed esophagus (Fig. 3I) were seen 1 year after revision surgery.

\section{Discussion}

Esophageal perforation may not be immediately identified in anterior cervical spine surgery; hence, a later possibility must be considered. The esophagus is located in the superficial layer of the deep cervical fascia, running on the back of the organ and surrounded by smooth muscle [3]. Although dysphagia is the most common symptom of 


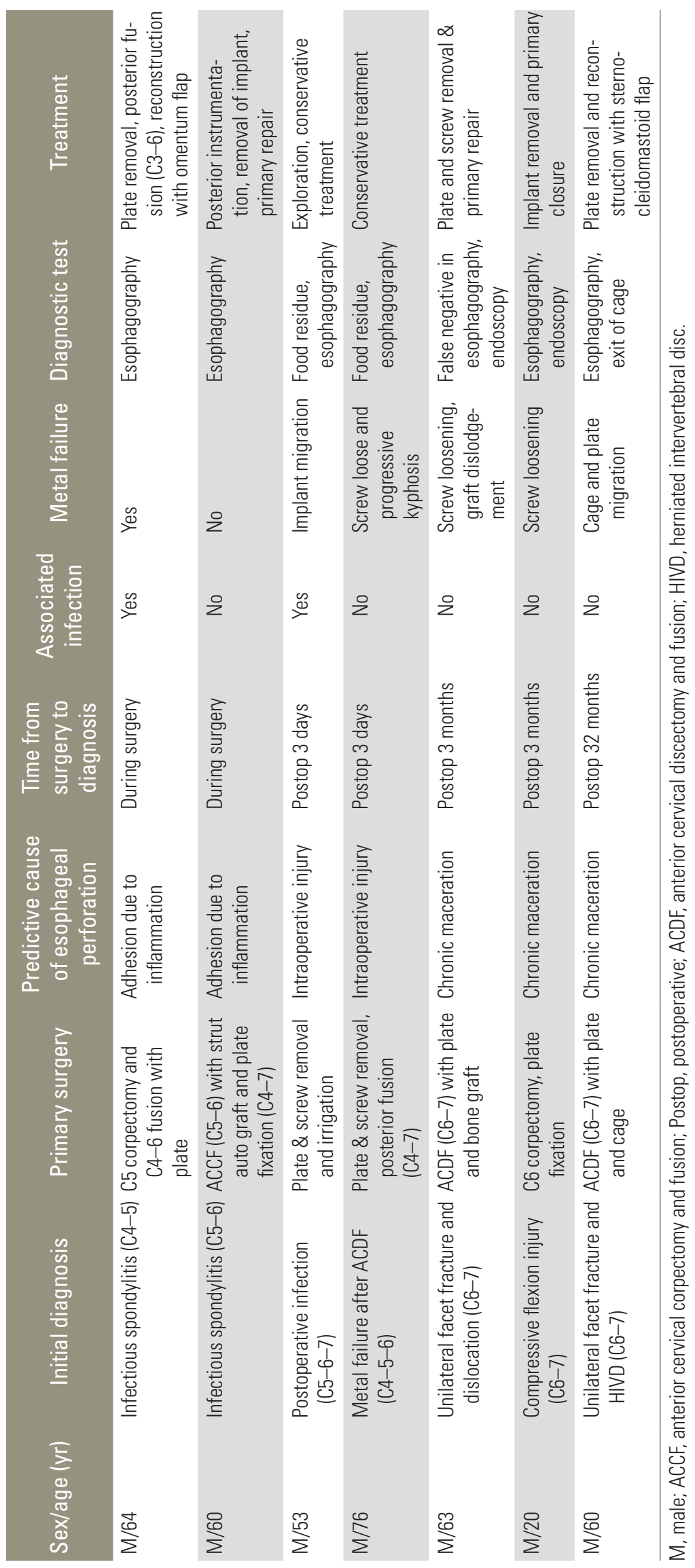



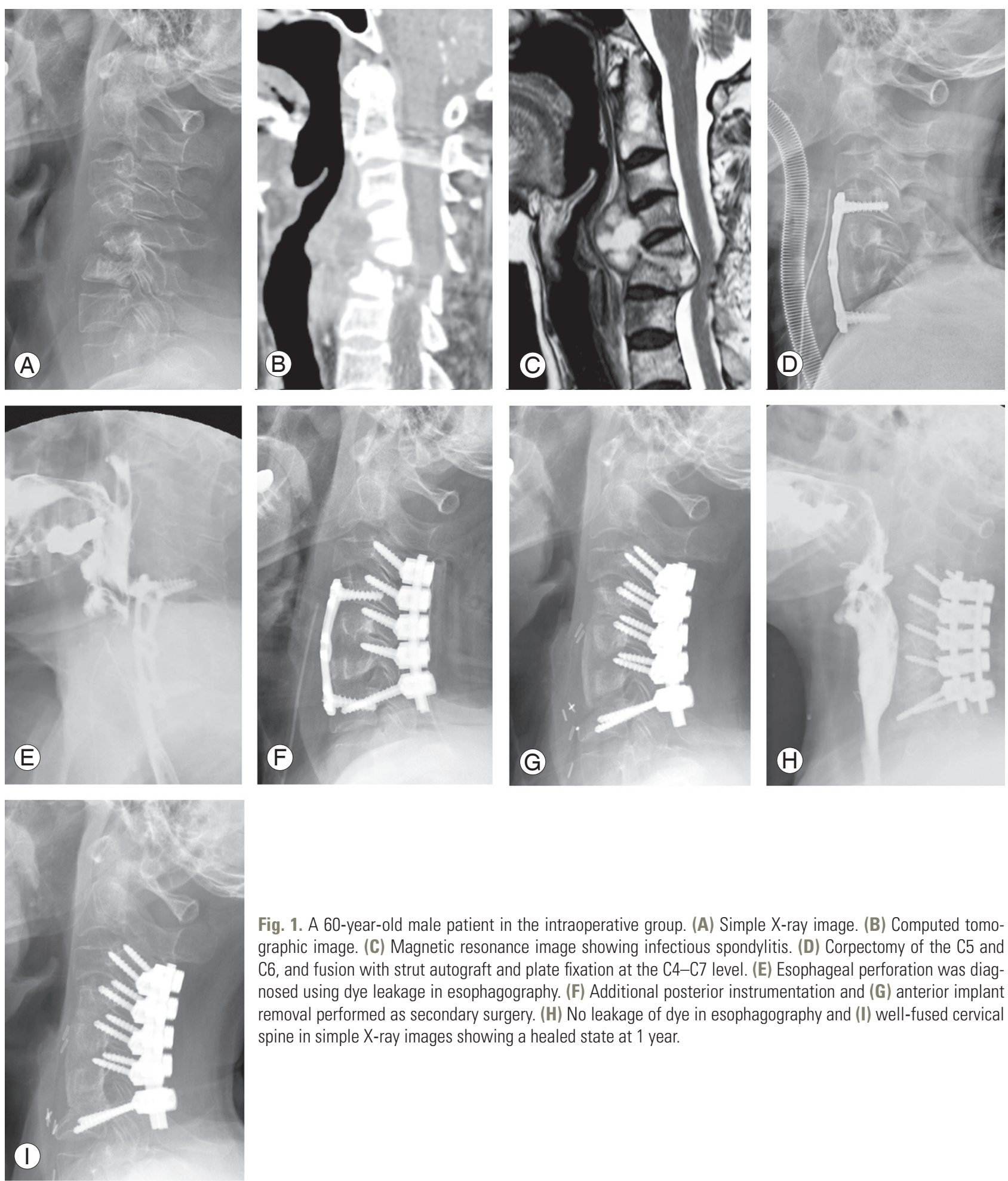

Fig. 1. A 60-year-old male patient in the intraoperative group. (A) Simple X-ray image. (B) Computed tomographic image. (C) Magnetic resonance image showing infectious spondylitis. (D) Corpectomy of the C5 and C6, and fusion with strut autograft and plate fixation at the C4-C7 level. (E) Esophageal perforation was diagnosed using dye leakage in esophagography. (F) Additional posterior instrumentation and (G) anterior implant removal performed as secondary surgery. (H) No leakage of dye in esophagography and (I) well-fused cervical spine in simple $\mathrm{X}$-ray images showing a healed state at 1 year.

esophageal perforation, other symptoms, such as pharyngeal pain, odynophagia, unexplained aspiration, chocking, fever, localized neck tenderness, induration, hemoptysis, and subcutaneous emphysema can also occur $[4,5]$. Food residue, such as rice leaking from the surgical incision, is a pathognomonic finding. In the present study, dysphagia was the main symptom in all patients, and two patients were diagnosed by food residue drainage performed 3 days postoperatively. The risk factors for esophageal perforation were surgery (reoperation of the cervical spine, 

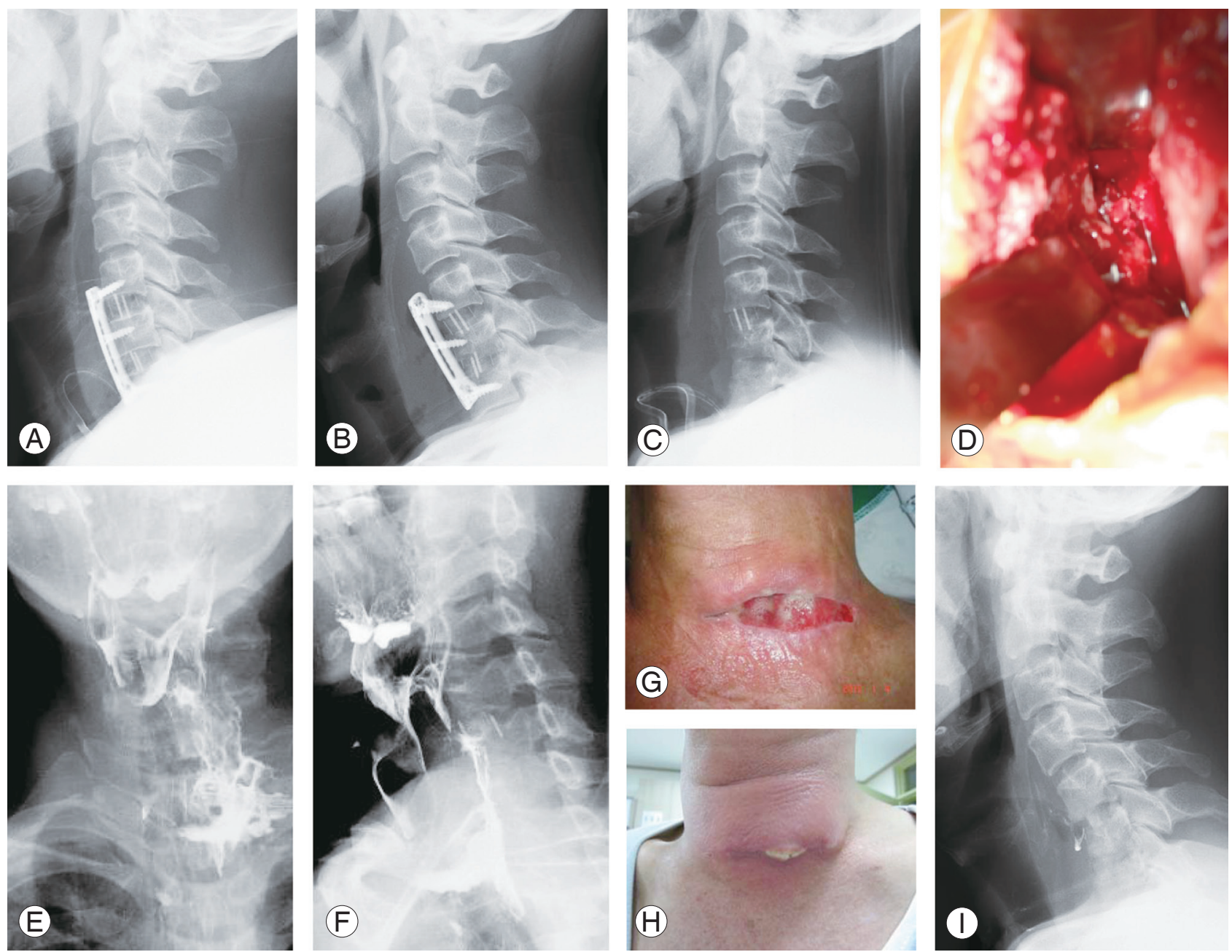

Fig. 2. A 53-year-old male patient in the perioperative group. (A) Anterior cervical discectomy and fusion at the C5-C7 as primary surgery. (B) Migrated plate and screw at 3 months, followed by infection. (C) Anterior plate and screw removal. (D) Esophageal perforation shown in exploration surgery. (E, F) Dye leakage in esophagography. (G) Food residue in wound site. (H) Healed wound. (I) Fused cervical spine after conservative treatment.

use of high-speed burr, use of sharp retractor, implant migration, and prolonged operative time) and patientrelated factors (diabetes mellitus or tumor). In this study, the cause of esophageal perforation, classified by time of onset, was infectious spondylitis in the intraoperative group, revision surgery (one case of spondylitis and one case of metal failure) in the perioperative group, and chronic maceration of metal after traumatic fracture surgery in the delayed group.

Secondary infections are another main symptom of esophageal perforation, but delayed infections because of esophageal perforation are not characterized by symptoms. Broad-spectrum antibiotics, which are sensitive to both gram-positive and -negative organisms, should be administered, even before the identification of bacteria
[6]. In this study, methicillin-resistance Streptococcus epidermidis was identified in one of the two patients in the intraoperative group. The patient eventually died of sepsis caused by uncontrolled infection. Streptococcus epidermidis and S. viridans were identified in one of two patients in the perioperative group. The patients in the delayed group had no associated infections.

Plain neck radiographs may be helpful in visualizing indirect signs of perforation, such as the presence of prevertebral air, subcutaneous emphysema, widening of the retropharyngo-esophageal space, and migration of cervical implants. Barium esophagogram (contrast swallow study) may help confirm the diagnosis and locate the perforation, showing extravasation of fluid and/or air fluid collection in deep neck spaces [7]. Although esophagos- 

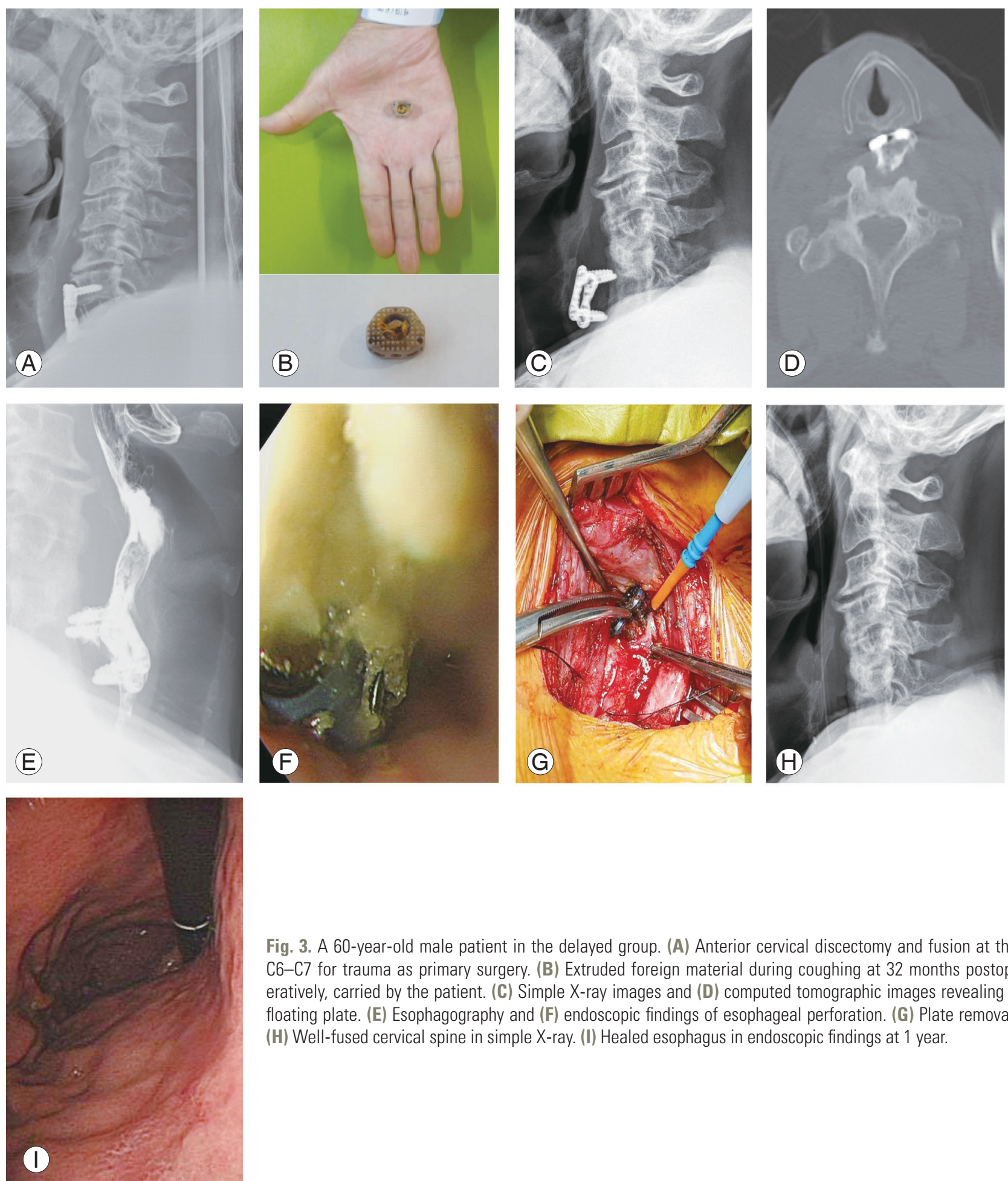

Fig. 3. A 60-year-old male patient in the delayed group. (A) Anterior cervical discectomy and fusion at the C6-C7 for trauma as primary surgery. (B) Extruded foreign material during coughing at 32 months postoperatively, carried by the patient. (C) Simple X-ray images and (D) computed tomographic images revealing a floating plate. (E) Esophagography and (F) endoscopic findings of esophageal perforation. (G) Plate removal. (H) Well-fused cervical spine in simple X-ray. (I) Healed esophagus in endoscopic findings at 1 year.

copy can provide direct visualization of the perforation, it may miss perforation hidden in a mucosal fold, leading to high false-negative rates $(10 \%-32 \%)$ [8]. In our patients, esophagography was the most common confirmative diagnostic method (six cases), but it produced a falsenegative result in one case. Endoscopic examination was the confirmative diagnostic method in three cases, with no false-negative findings. One case was confirmatively diagnosed by direct visualization through secondary exploration.

Management of esophageal perforation may vary based on the time of diagnosis, size and shape of injury, accom- 
panying surgical site infection, and the patient's general condition. Multidisciplinary cooperation between otolaryngologists, gastroenterologists, spine surgeons, and cardiothoracic surgeons is essential. The mortality rate is $20 \%$ if treated within 24 hours, but is increased to $50 \%$ if delayed $>24$ hours $[9,10]$. Small perforation, a wellcontained leak with no sign of sepsis, is only possible with conventional treatment $[11,12]$. Conventional therapy requires prohibition of oral administration for at least 1 week, administration of broad-spectrum antibiotics, administration of prokinetic drugs (cisapride and metoclopramide), and feeding via nasogastric tube [11]. However, $20 \%-25 \%$ of patients develop abscesses because of treatment failure even with conventional treatment, resulting in a mortality rate of $18 \%[10,13]$.

Primary closure of perforation and prevention of infection is the gold standard of treatment if a surgical procedure is required [13]. If primary closure is impossible, sternocleidomastoid muscle flap, pectoralis major muscle flap, or a longus coli muscle flap can be reinforced after a double-layer or imbricating suture. Supportive treatment, in addition to surgical treatment, is also important. Jejunostomy is recommended when the nasogastric tube is maintained for at least two weeks [11]. In the intraoperative group, anterior plate and screw removal and posterior instrumentation were performed. Esophageal perforation was treated with reconstruction using the omentum flap in one patient and primary repair in one patient. Esophageal perforation was treated conservatively after implant removal surgery in all patients in the perioperative group. Anterior plate and screw removal was performed in all three patients in the delayed group. Esophageal perforation was treated by primary closure in two patients who were diagnosed 3 months postoperatively. Esophageal perforation was treated by reconstruction using the sternocleidomastoid muscle flap in a patient who was diagnosed 32 months postoperatively. Drainage was performed in all cases, and the mean duration of drainage was 5.6 days (range, 2-12 days). One patient developed sepsis postoperatively, followed by disseminated intravascular coagulation, and died. The remaining six patients were cured without serious neurologic sequelae.

The retrospective, multicenter design of the present study is a fundamental limitation. Our conclusions may not be strongly appealing to other spine surgeons and practitioners. We understand that the drawback of our study is inevitable because esophageal perforation is a rare complication occurring after anterior cervical spine surgery.

\section{Conclusions}

The main cause of intraoperative esophageal perforation was esophageal adhesions because of infectious spondylitis. However, perioperative and delayed esophageal perforations were caused by chronic irritation of metal failure. Anterior plate and screw removal is necessary, and posterior instrumentation and fusion may be considered in these patients, depending on the fusion status. Repair method for esophageal perforation should be determined by considering the severity of esophageal perforation.

\section{Conflict of Interest}

No potential conflict of interest relevant to this article was reported.

\section{References}

1. Fisichella PM, Soper NJ, Pellegrini CA, Patti MG. Surgical management of benign esophageal disorders: the "Chicago approach." London: SpringerVerlag; 2014.

2. Fountas KN, Kapsalaki EZ, Nikolakakos LG, et al. Anterior cervical discectomy and fusion associated complications. Spine (Phila Pa 1976) 2007;32:2310-7.

3. Lu X, Guo Q, Ni B. Esophagus perforation complicating anterior cervical spine surgery. Eur Spine J 2012;21:172-7.

4. Anandaswamy TC, Pujari VS, Shivanna S, Manjunath A. Delayed pharyngoesophageal perforation following anterior cervical spine surgery: an incidental finding. J Anaesthesiol Clin Pharmacol 2012;28:13940.

5. Gaudinez RF, English GM, Gebhard JS, Brugman JL, Donaldson DH, Brown CW. Esophageal perforations after anterior cervical surgery. J Spinal Disord 2000;13:77-84.

6. Lew TW, Darby J, Marion DW. Candida mediastinitis and septic shock following occult esophageal perforation in a patient with posttraumatic quadriplegia. J Trauma 1995;39:805-8.

7. Brinster CJ, Singhal S, Lee L, Marshall MB, Kaiser LR, Kucharczuk JC. Evolving options in the manage- 
ment of esophageal perforation. Ann Thorac Surg 2004;77:1475-83.

8. Ardon $\mathrm{H}$, van Calenbergh F, van Raemdonck D, et al. Oesophageal perforation after anterior cervical surgery: management in four patients. Acta Neurochir (Wien) 2009;151:297-302.

9. Pompili A, Canitano S, Caroli F, et al. Asymptomatic esophageal perforation caused by late screw migration after anterior cervical plating: report of a case and review of relevant literature. Spine (Phila $\mathrm{Pa}$ 1976) 2002;27:E499-502.

10. Orlando ER, Caroli E, Ferrante L. Management of the cervical esophagus and hypofarinx perforations complicating anterior cervical spine surgery. Spine (Phila Pa 1976) 2003;28:E290-5.

11. Gupta NM, Kaman L. Personal management of 57 consecutive patients with esophageal perforation. Am J Surg 2004;187:58-63.

12. Kim YJ, Glazer PA. Delayed esophageal perforation and abscess formation after cervical vertebrectomy and fusion. Orthopedics 2002;25:1091-3.

13. Navarro R, Javahery R, Eismont F, et al. The role of the sternocleidomastoid muscle flap for esophageal fistula repair in anterior cervical spine surgery. Spine (Phila Pa 1976) 2005;30:E617-22. 